Artigo Original

Original Article

Haydée Fiszbein Wertzner ${ }^{1}$

Luciana de Oliveira Pagan-Neves²

Renata Ramos Alves ${ }^{2}$

Tatiane Faria Barrozo ${ }^{2}$

Descritores

Patologia da fala e linguagem

Fala

Testes de articulação da fala

Medida da produção da fala

Criança

Keywords

Speech-language pathology

Speech

Speech articulation tests

Speech production measurement

Child

Endereço para correspondência:

Haydée Fiszbein Wertzner

R. Cipotânea, 51, Cidade Universitária, Butantã, São Paulo (SP), Brasil, CEP: 05360-160.

E-mail: hfwertzn@usp.br

Recebido em: 3/8/2011

Aceito em: 6/8/2012

\section{Implicações da diadococinesia oral no transtorno fonológico}

\author{
Implications of diadochokinesia in children with speech \\ sound disorder
}

\section{RESUMO}

Objetivo: Verificar o desempenho de crianças com e sem transtorno fonológico nas habilidades motoras orais medidas pela diadococinesia oral, de acordo com as variáveis idade e gênero, além de comparar os métodos de análise manual e por meio de software específico. Métodos: Participaram do estudo 72 sujeitos, com idade entre 5 anos e 7 anos e 11 meses, que foram divididos em quatro subgrupos em função da presença do diagnóstico de transtorno fonológico (grupo controle e grupo estudo) e da idade ( $\leq 6$ anos e 5 meses e $>6$ anos e 5 meses). A diadococinesia oral foi avaliada na repetição das sequências 'pa', 'ta', 'ka' e 'pataka' utilizando o método de análise manual e pelo software Motor Speech Profile ${ }^{\circledast}$. Resultados: Houve diferença somente relacionada à variável gênero nos grupos, mas que não influenciou na produção do número de sequências por segundo. Houve correlação entre o número de sequências por segundo e a idade em todas as sequências, exceto para 'ka', somente no grupo controle. Os resultados da comparação entre grupos indicaram que não houve diferença entre as distribuições do número de sequências por segundo nas sequências estudadas nas duas faixas etárias analisadas. Observou-se concordância forte entre as medidas realizadas a partir dos dois métodos. Conclusão: O presente estudo mostrou a importância de se avaliar funcionalmente, por meio da aplicação de diferentes métodos de análise, aspectos do processamento motor da fala em crianças com transtorno fonológico, evidenciando as dificuldades motoras em crianças na faixa etária inferior a 8 anos de idade.

\begin{abstract}
Purpose: To verify the performance of children with and without speech sound disorder in oral motor skills measured by oral diadochokinesia according to age and gender and to compare the results by two different methods of analysis. Methods: Participants were 72 subjects aged from 5 years to 7 years and 11 months divided into four subgroups according to the presence of speech sound disorder (Study Group and Control Group) and age ( $\leq 6$ years and 5 months and $>6$ years and 5 months). Diadochokinesia skills were assessed by the repetition of the sequences 'pa', 'ta', 'ka' and 'pataka' measured both manually and by the software

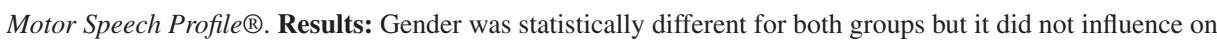
the number of sequences per second produced. Correlation between the number of sequences per second and age was observed for all sequences (except for ' $\mathrm{ka}$ ') only for the control group children. Comparison between groups did not indicate differences between the number of sequences per second and age. Results presented strong agreement between the values of oral diadochokinesia measured manually and by MSP. Conclusion: This research demonstrated the importance of using different methods of analysis on the functional evaluation of oro-motor processing aspects of children with speech sound disorder and evidenced the oro-motor difficulties on children aged under than eight years old.
\end{abstract}

Trabalho realizado no Curso de Fonoaudiologia, Departamento de Fisioterapia, Fonoaudiologia e Terapia Ocupacional, Faculdade de Medicina, Universidade de São Paulo - USP - São Paulo (SP), Brasil.

(1) Departamento de Fisioterapia, Fonoaudiologia e Terapia Ocupacional, Faculdade de Medicina, Universidade de São Paulo - USP - São Paulo (SP), Brasil.

(2) Laboratório de Investigação Fonoaudiológica da Aquisição Fonológica e do Distúrbio Fonológico, Curso de Fonoaudiologia, Faculdade de Medicina, Universidade de São Paulo - USP - São Paulo (SP), Brasil.

Conflito de interesses: Não 


\section{INTRODUÇÃO}

As diferentes causas, a heterogeneidade de manifestação e a gravidade do transtorno fonológico (TF) na população infantil ainda são alvos de diversos questionamentos, os quais envolvem principalmente os aspectos relacionados ao diagnóstico do TF.

A avaliação para o diagnóstico do TF apoia-se em provas de imitação de palavras, nomeação de figuras e fala espontânea que possibilitam a verificação de alterações fonológicas e o levantamento do inventário fonético. Apesar da importância dessas provas para a constatação da presença do TF existe a necessidade de se complementar o diagnóstico com provas que forneçam informações mais detalhadas para cada caso e que auxiliem para a posterior escolha do modelo terapêutico mais adequado.

Assim, diagnosticar um indivíduo como portador de TF, envolve a aplicação de diversas provas nas quais se procura estabelecer além da gravidade (que pode ser medida por diferentes índices $)^{(1)} \mathrm{o}$ aspecto mais afetado em cada indivíduo: a percepção auditiva, o processamento cognitivo-linguístico ou o processamento motor da fala ${ }^{(2-4)}$. Os erros de fala apresentados pela criança durante o período de desenvolvimento de linguagem podem estar relacionados ao planejamento e à execução de movimentos motores finos que são necessários para a produção articulatória dos sons ${ }^{(3)}$. A integração entre esses processamentos é responsável pela emergência das primeiras palavras na língua e pelo conhecimento fonológico.

A avaliação da diadococinesia oral (DDK) fornece evidências que auxiliam na verificação da relação existente entre as alterações de fala e as habilidades motoras orais. Assim, a DDK é um procedimento que complementa o diagnóstico do TF, uma vez que mede a capacidade de revezar ações musculares opostas, por meio de movimentação rápida, de maneira alternada e repetida dos órgãos articuladores ${ }^{(3,5-7)}$ avaliando, desta forma, a evolução da maturação e da integração neuromotora do indivíduo.

A mensuração da DDK pode ser realizada de várias maneiras, tanto no que diz respeito ao estímulo empregado para coleta como no método de análise. De forma geral, o estímulo mais observado é a repetição de sílabas isoladas ou de um conjunto de sílabas sem significado durante um determinado período de tempo ${ }^{(8)}$. As mais citadas na literatura, tanto para a língua inglesa ${ }^{(5,6,8)}$, como para o Português Brasileiro ${ }^{(9-13)}$, são os monossílabos pa, ta, ka e o trissílabo pataka.

Uma das maiores dificuldades em se comparar os resultados dos diferentes estudos, no que diz respeito aos valores do número de repetições durante um determinado período de tempo obtidos durante a aplicação da DDK, deve-se ao fato da variabilidade na coleta dos dados descrita no método ${ }^{(14)}$.

A DDK pode ser medida manualmente ou com o auxílio de um programa de análise acústica. Na literatura, verifica-se que alguns estudos utilizam a medida manual para contar as sequências produzidas pelo indivíduo em intervalo de tempo pré-determinado ${ }^{(9,14)}$, para contar o tempo que o indivíduo leva para realizar um determinado número de repetições ${ }^{(15)}$ enquanto outros descrevem ainda as medidas de acordo com as duas formas de análise ${ }^{(7)}$. A medida manual é de fácil utilização para o fonoaudiólogo clínico, e pode ser usada desde que sejam tomados cuidados em relação à coleta e análise das sequências.

Já a medição da DDK feita com o auxílio da análise acústica oferece maior segurança já que possibilita o registro dos valores obtidos por meio do software utilizado. Além de quantificar o número de sequências produzidas, de acordo com o equipamento utilizado é possível obter outras medidas. No Motor Speech Profile (MSP), modelo 5141 fabricado pela KayPentax ${ }^{\circledR}$ que é um software opcional para ser acoplado ao Computerized Speech Lab (CSL), há a possibilidade de serem obtidas informações a respeito do desvio padrão, o coeficiente de variação, o índice de variação entre os ciclos de repetição e outras medidas relacionadas ao pico de intensidade.

Um estudo recentemente publicado sobre a análise da DDK realizada com o auxílio do MSP em crianças com desenvolvimento normal de fala e linguagem falantes do Português Brasileiro e idade compreendida entre 8 e 10 anos, indicou que a média do número de sequências por segundo variou entre 4,8 e 5,2 para a sílaba 'pa', entre 4,9 e 5,4 para 'ta' e entre 4,3 e 4,9 para ' $\mathrm{ka}{ }^{\prime}(13)$.

Outro estudo ${ }^{(15)}$ realizado com a medida manual avaliando tanto crianças em desenvolvimento normal de fala e linguagem como as com TF, na faixa etária de 5 a 10 anos, apontou o uso da análise da DDK, através das repetições do trissílabo 'pataka', como um teste com alto poder discriminatório para confirmação do diagnóstico fonoaudiológico de TF em crianças com idade superior a 8 anos. $\mathrm{O}$ valor de corte encontrado pelo estudo que separou os sujeitos com e sem diagnóstico de TF foi de 1,4 sequências por segundo.

Assim, baseados na literatura, as hipóteses que motivaram a realização deste estudo foram: (1) as crianças sem alterações de fala e linguagem apresentam maior número de sequências por segundo na prova DDK oral; (2) não há diferença entre a medida da DDK oral feita manualmente ou com o auxílio do MSP; (3) o desempenho no número de sequências por segundo está associado à idade.

Assim, o objetivo deste estudo foi verificar o desempenho de crianças com e sem transtorno fonológico nas habilidades motoras orais medidas pela DDK, de acordo com as variáveis idade e gênero, além de comparar os métodos de análise manual e o realizada por meio de software específico.

\section{MÉTODOS}

A pesquisa foi aprovada pela Comissão de Ética para Análise de Projetos de Pesquisa (CAPPesq) da Diretoria Clínica do Hospital das Clínicas e da Faculdade de Medicina da Universidade de São Paulo (FMUSP) (nº 0958/08). Todos os responsáveis assinaram o Termo de Consentimento Livre e Esclarecido (TCLE).

Participaram desta pesquisa 72 crianças, sendo 36 sem queixas de alteração de fala e linguagem (GC), e 36 crianças apresentando transtorno fonológico (GE) com idade entre 5 anos e 7 anos e 11 meses de ambos os gêneros. O pareamento dos sujeitos quanto á idade e gênero não foi possível devido às perdas amostrais principalmente relacionadas às ausências dos pacientes durante o processo de avaliação. 
O critério de inclusão do GC foi não apresentar queixas (pais e professores) de alterações de audição, fala e linguagem, ter desempenho adequado na prova de fonologia ${ }^{(16)}$ do Teste de Linguagem Infantil $\mathrm{ABFW}^{(17)}$ e fala espontânea a partir do livro de história infantil Esconde-Esconde ${ }^{(18)}$.

Para o GE o critério de inclusão foi apresentar o diagnóstico de transtorno fonológico estabelecido em um laboratório fonoaudiológico específico após terem sido aplicadas as provas diagnósticas de fonologia e a anamnese aos pais.

Para a coleta dos dados da DDK foi utilizado o Computerized Speech Lab (CSL) 4500 da Kay-Pentax ${ }^{\circledR}$ acoplado ao microfone de alta performance e fidelidade da Kay-Pentax ${ }^{\circledR}$ modelo 3706. Para a gravação das provas de fonologia e fala espontânea, foi utilizado o gravador ZoomH2 Handy recorder e o microfone Vocal Evolution 817 Sennheiser. A coleta foi realizada em uma sala acusticamente tratada e todas as repetições da DDK foram arquivadas em um computador.

A verificação do número de sequências produzidas no período de tempo pré-determinado foi realizada de duas maneiras: manualmente a partir da visualização das repetições das sequências solicitadas por meio do espectrograma obtido no CSL, e por meio do software Motor Speech Profile ${ }^{\circledR}$ (MSPmodelo 5141).

\section{Aplicação das provas experimentais}

Para a coleta dos dados da DDK, os participantes foram solicitados a repetir as sequências monossilábicas 'pa', 'ta', 'ka' e a sequência trissilábica 'pataka' durante 10 segundos. As crianças foram instruídas a repetir a sequência solicitada o mais rápido possível durante dez segundos (enquanto o braço da avaliadora permanecesse levantado). Os 10 segundos foram contabilizados a partir do início da produção da sequência solicitada, sendo que a duração das eventuais pausas (respiratórias ou referentes a algum comentário) foi desconsiderada para o cálculo do tempo de análise. Para o treino realizado antes da coleta da amostra definitiva foi utilizada a repetição da sequência 'badaga'. As sequências foram coletadas em diferentes ordens para evitar possíveis vieses de aplicação da prova.

A análise da DDK foi feita manualmente nas amostras de fala dos 36 sujeitos do GC e 36 do GE. Na análise realizada por meio do software MSP foram estudadas somente as amostras de fala de 15 crianças do GC e 12 do GE, em função da ocorrência de perdas amostrais.

As perdas amostrais registradas ocorreram principalmente como consequência da dificuldade na coleta de dados acústicos que requerem concentração da criança e estabilidade dos equipamentos e, por isso, a gravação destes dados é feita diretamente no equipamento (CSL e software MSP) que fica alocado no laboratório onde a pesquisa foi realizada. No GC tais perdas foram decorrentes do não comparecimento à sessão agendada para coleta dos dados da DDK que foram analisados por meio do software MSP. Em relação ao GE, a diferença no número de análises realizadas ocorreu devido às interferências no momento da coleta que impossibilitaram a análise dos dados, tais como: o registro da fala da criança em intensidade não suficiente para ser captada pelo programa e gravações que foram encobertas por ruídos como batidas no microfone e soltura de ar ruidoso.

\section{Análise manual}

No modo manual, para cada sequência 'pa', 'ta', 'ka' e 'pataka' foi calculado o número de sequências por segundo, obtido a partir do número de sequências realizadas pelas crianças, dividido pela duração do tempo de emissão. Para esta análise foi desconsiderado o tempo inicial de três segundos e do tempo final de dois segundos, restando assim os cinco segundo mediais para análise. A contagem do número de sequências por segundo foi realizada por meio auditivo, com o apoio visual do espectrograma gerado pelo CSL no tempo selecionado (cinco segundos). Desta forma, no presente estudo, o cálculo do número de sequências por segundo foi feito dividindo-se o número de sequências realizadas por cinco segundos.

\section{Análise pelo MSP}

O software MSP analisa e extrai parâmetros relevantes para fala com o intuito de averiguar a dificuldade motora. Conforme descrito no manual do MSP ${ }^{(19)}$, a análise realizada mede a habilidade do sujeito em repetir a combinação consoante-vogal de modo rápido, constante e ritmado $\mathrm{O}$ software pode gerar automaticamente e simultaneamente 11 diferentes parâmetros de análise, sendo que no presente estudo foi registrado a DDKavr, que mede em segundos o número médio de repetições.

A análise realizada no MSP contou com oito segundos, sendo desprezado o primeiro e o último segundo da gravação. A escolha do tempo de oito segundos apoiou-se nas instruções do manual MSP(19) que considera essa uma duração adequada para a extração das medidas.

\section{Método estatístico}

A distribuição dos sujeitos participantes do estudo de acordo com a variável idade foi feita por meio do teste T-student e, para a variável gênero, pelo teste do qui-quadrado. O número de sequências por segundo produzido pelas crianças dos dois grupos foi comparado pelo teste de Mann-Whitney. A correlação de Spearman foi aplicada entre os valores de cada prova e a idade. Nos casos em que não houve correlação os grupos, GC e GE, foram comparados pelo teste t-Student.

Quando foi detectada a presença de significância indicando correlação entre os valores da prova e a idade os, grupos (GC e $\mathrm{GE}$ ) foram subdivididos em função da idade. Considerando que a mediana da idade dos grupos foi de 6 anos e 5 meses os novos agrupamentos foram: $\leq 6$ anos e 5 meses e $>6$ anos e 5 meses. A combinação dos grupos com as faixas etárias deu origem a quatro subgrupos: GC com idade $\leq 6$ anos e 5 meses, GC com idade $>6$ anos e 5 meses, GE com idade $\leq 6$ anos e 5 meses e GE com idade $>6$ anos e 5 meses. O teste de Kruskal-Wallis foi utilizado para comparar os quatro subgrupos. Quando houve necessidade os valores de $\mathrm{p}$ foram corrigidos pelo procedimento de Bonferroni. Para medir a concordância das medidas da 
DDK obtidas pelo método manual e pelo MSP foi calculado o coeficiente de correlação intraclasse.

\section{RESULTADOS}

Caracterização dos sujeitos de acordo com idade e gênero

A análise comparativa entre os sujeitos dos dois grupos de acordo com as variáveis idade e gênero pode ser observada na Tabela 1. Houve diferença somente relacionada à variável gênero em cada um dos grupos, mas não em relação à idade.

Tabela 1. Análise comparativa entre grupos para as variáveis idade e gênero.

\begin{tabular}{|c|c|c|c|}
\hline \multirow{2}{*}{ Grupo } & \multicolumn{2}{|c|}{ Idade } & \multirow{2}{*}{ Valor de $p$} \\
\hline & $\mathrm{n}$ & Média & \\
\hline GC & 36 & 6,4 & \multirow{2}{*}{0,935} \\
\hline GE & 36 & 6,4 & \\
\hline \multirow{2}{*}{ Grupo } & \multicolumn{2}{|c|}{ Gênero } & \multirow{2}{*}{ Valor de $p$} \\
\hline & Feminino & Masculino & \\
\hline GC & 22 & 14 & \multirow{2}{*}{$0,009^{*}$} \\
\hline GE & 11 & 25 & \\
\hline
\end{tabular}

*Valores significativos $(p \leq 0,05)$ - Testes T Student e Qui-quadrado

Legenda: $\mathrm{GE}$ = grupo de estudo; $\mathrm{GC}$ = grupo controle

\section{Desempenho na DDK oral pela análise manual}

Para a análise manual da DDK, que contou com 36 sujeitos em cada um dos grupos foi realizada a comparação intragrupo para os gêneros em cada uma das sequências estudadas. Na Tabela 2 são apresentados os p-valores obtidos. Não houve diferenças entre gêneros e o número de sequências por segundo.

Tabela 2. Valores de pobtidos no teste de igualdade das distribuições do número de sequências por segundo nos dois gêneros em cada um dos grupos.

\begin{tabular}{lcc}
\hline Sequência & GC & GE \\
\hline 'pa' & 0,363 & 0,891 \\
'ta' & 0,372 & 0,642 \\
'ka' & 0,697 & 0,594 \\
'pataka' & 0,108 & 0,887 \\
\hline
\end{tabular}

Teste de Mann-Whitney $(p<0,05)$

Legenda: GE = grupo de estudo; GC = grupo controle

No GE não houve evidência de correlação entre o número de sequências por segundo medidas manualmente e a idade em nenhuma das sequências. Já no GC, houve correlação em todas as sequências, exceto para 'ka' (Tabela 3).

A comparação entre grupos (teste de Mann-Whitney), do número de sequências por segundo para a repetição da sequência da sílaba 'ka', não indicou diferença $(\mathrm{p}=0,478)$. O teste de Kruskal-Wallis foi aplicado para comparar as distribuições do número de sequências por segundo entre GC e GE na repetição das outras sequências nas quais foi observada significância estatística para a idade dos sujeitos do GC. Assim, os sujeitos
Tabela 3. Correlação entre o número de sequências por segundo medido manualmente e a idade.

\begin{tabular}{llcc}
\hline Sequência & & $\mathrm{GC}$ & $\mathrm{GE}$ \\
\hline \multirow{2}{*}{ 'pa' } & $\mathrm{R}$ & 0,537 & $-0,075$ \\
& Valor de $\mathrm{p}$ & $0,001^{*}$ & 0,663 \\
& $\mathrm{n}$ & 36 & 36 \\
\hline \multirow{2}{*}{ 'ta' } & $\mathrm{R}$ & 0,549 & 0,191 \\
& Valor de $\mathrm{p}$ & $0,001^{*}$ & 0,265 \\
& $\mathrm{n}$ & 36 & 36 \\
\hline \multirow{2}{*}{ 'ka' } & $\mathrm{R}$ & 0,297 & 0,010 \\
& $\mathrm{~V}$ & 0,079 & 0,954 \\
& $\mathrm{n}$ & 36 & 36 \\
\hline \multirow{2}{*}{ 'pataka' } & $\mathrm{R}$ & 0,413 & 0,183 \\
& Valor de $\mathrm{p}$ & $0,012^{*}$ & 0,291 \\
& $\mathrm{n}$ & 36 & 35 \\
\hline
\end{tabular}

*Valores significativos $(p \leq 0,05)$ - Coeficiente de Correlação de Spearman

Legenda: $\mathrm{GE}=$ grupo de estudo; $\mathrm{GC}=$ grupo controle; $\mathrm{R}=$ correlação

do GC e do GE foram reagrupados em dois subgrupos de acordo com a idade ( $\leq 6$ anos e 5 meses e $>6$ anos e 5 meses). Os resultados da comparação entre grupos indicaram que não houve diferença entre as distribuições do número de sequências por segundo nas sequências 'pa', 'ta' e 'pataka' tanto na faixa etária $\leq 6$ anos e 5 meses para 'pa' $(\mathrm{p}=0,078)$, 'ta' $(\mathrm{p}=0,396)$ e 'pataka' ( $>0,999)$, quanto na faixa etária $>6$ anos e 5 meses para 'pa' $(p>0,999)$, 'ta' $(p=0,408)$ e 'pataka' $(p=0,078)$.

\section{Comparação entre a análise da DDK manual e a realizada no MSP}

A análise da concordância entre as medidas de sequências por segundo feitas manualmente e pelo MSP foi feita somente para a as sílabas isoladas 'pa', 'ta', 'ka', uma vez que o programa somente analisa tais sequências. Os dados descritivos das duas análises encontram-se na Tabela 4.

Para medir a concordância das medidas obtidas pelos dois métodos foi calculado o coeficiente de correlação intraclasse (Tabela 5). Os resultados indicaram que nas sequências 'pa', 'ta' e ' $k a$ ' o valor observado do coeficiente foi maior que 0,9 indicando concordância forte entre as medidas dos dois métodos.

\section{DISCUSSÃO}

A heterogeneidade do TF em relação aos fatores causais, a manifestação e a gravidade ainda são muito estudados. Aplicar provas complementares às específicas de fonologia contribui para o melhor entendimento das manifestações do TF. Tais provas complementares devem buscar esclarecer possíveis comprometimentos dos processamentos da linguagem, motor da fala e da audição, bem como na interação entre esses processamentos.

A medida da DDK oral, utilizada no presente estudo, como prova complementar ao diagnóstico do TF, pode contribuir com evidências a respeito da programação motora da fala ${ }^{(10,20)}$. A 
Tabela 4. Estatística descritiva para o número de sequências por segundo na prova de DDK analisada pelo MSP e manualmente

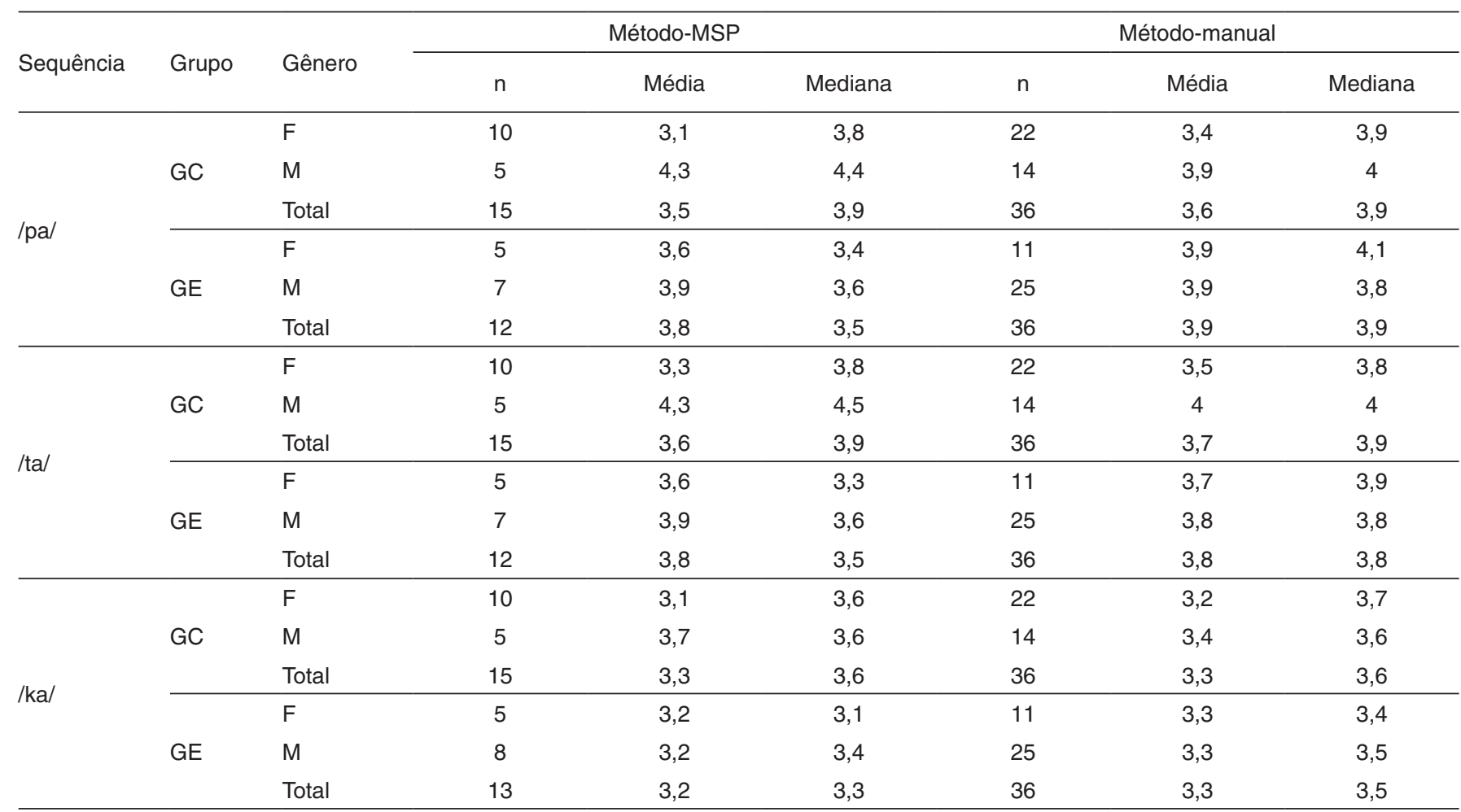

Legenda: $\mathrm{GE}=$ grupo de estudo; $\mathrm{GC}=$ grupo controle; $\mathrm{F}=$ feminino; $\mathrm{M}=$ masculino

Tabela 5. Concordância entre o número de sequências por segundo obtido a partir da análise manual e do MSP.

\begin{tabular}{lcc}
\hline Sequência & Coeficiente & Intervalo de confiança de 95\% \\
\hline 'pa' & 0,94 & {$[0,88 ; 0,97]$} \\
'ta' & 0,91 & {$[0,80 ; 0,96]$} \\
'ka' & 0,95 & {$[0,87 ; 0,98]$} \\
\hline
\end{tabular}

Coeficiente de correlação intraclasse

aplicação e análise da DDK dependem de vários fatores como a idade dos sujeitos, a forma de coleta de dados (ordem dada ao sujeito), tempo de análise das sequências, tipo de sequência analisada e do tratamento acústico dado ao sinal gravado para análise. Essas questões aparecem de forma variada nos estudos publicados na literatura ${ }^{(5,9,10,12,14,15,21,22)}$, o que dificulta comparações.

No presente estudo, a análise da DDK indicou que, apesar da diferença de gênero entre os dois grupos, não houve diferença entre a variável gênero e o número de sequências por segundo em nenhuma das sequências analisadas, tanto no GC quanto no GE. É importante destacar que outro estudo realizado com sujeitos em desenvolvimento normal de fala ${ }^{(13)}$ apontou desempenho diferente na DDK entre os gêneros (somente na sequência trissilábica pataka e no monossílabo ta), porém a idade estudada foi superior à das crianças participantes desta pesquisa. Portanto, a presente pesquisa evidencia que o desempenho na DDK na faixa etária estudada é semelhante nos dois gêneros.

A literatura ${ }^{(23-28)}$ mostra que a habilidade motora da fala começa a ser refinada a partir dos 8 anos estendendo-se até os 16 anos e, portanto, as crianças com idade inferior a esta não apresentam controle neuromuscular para produzir os ajustes motores necessários para a produção adequada de todas as sequências sonoras e, consequentemente, precisam adotar estratégias para se aproximar do modelo adulto de fala ${ }^{(28)}$. Como a faixa etária pesquisada neste estudo foi inferior aos 8 anos de idade, acredita-se que, antes desta idade as crianças ainda não possuem a maturação motora da fala necessária para que a articulação dos sons ocorra com maior precisão e velocidade ${ }^{(15)}$, sugerindo ainda que as crianças com idade de até 7 anos e 11 meses apresentem aspectos maturacionais do controle motor da fala semelhantes, independentemente do gênero.

Outro aspecto interessante é o fato de haver correlação entre a idade e o número de sequências por segundo na medida manual da DDK para as crianças do GC, mas não para as crianças do GE. Essa ausência de correlação no GE já poderia indicar uma maior dificuldade na maturação e na integração neuromotora das crianças com TF que não apresentam aumento no número de sequências conforme aumenta a idade, embora as comparações entre os grupos em função da idade não tenha mostrado diferença ${ }^{(29)}$.

É importante destacar que para a sequência 'pataka' a comparação entre as crianças dos dois grupos com idade superior a 6 anos e 5 meses mostrou uma tendência à significância. Isso indica que a sequência 'pataka' parece fornecer mais informações a respeito dos movimentos rápidos e repetidos envolvidos na fala e que, quanto maior a idade da criança, mais fidedigna é essa informação.

$\mathrm{Na}$ repetição da sequência 'pataka' o número médio de repetições do GC foi sempre maior que do GE. Este dado, apesar de não ter sido estatisticamente significante, sugere 
que, em algumas crianças com TF, ocorra uma dificuldade na habilidade motora revelada no controle de movimentos articulatórios de sequências. Tal fato relaciona-se ao maior nível de controle motor demandado por sequências polissilábicas quando comparada as sequências monossilábicas. Um maior número de sílabas implica num maior número de gestos articulatórios e programações motoras ${ }^{(10,13)}$ o que pode interferir nas dificuldades dos sujeitos com TF.

A questão da idade e do tipo de sequência também foi discutido em outro estudo ${ }^{(10,15)}$ no qual foi demonstrado que a prova DDK, por meio da sequência 'pataka', foi capaz de separar os grupos com e sem TF a partir de 8 anos de idade com alta sensibilidade e especificidade.

Em relação à comparação entre as duas formas de se analisar a prova DDK oral (manual e pelo MSP), obteve-se forte concordância entre os resultados, indicando que as duas medidas são bastante fidedignas. Dessa forma o modo de análise manual da prova DDK pode ser utilizado como uma opção tão confiável quanto a analise do software MSP, desde que seguidos os critérios propostos. Porém é importante destacar que para a análise manual foram estabelecidas algumas regras (descritas no método) que podem ter influenciado esse resultado. Assim, é recomendável que ao aplicar a avaliação e ao analisar a DDK essas recomendações sejam consideradas, para que não ocorram vieses.

A medida da DDK aplicada na presente pesquisa mostrou que há uma tendência das crianças com TF a produzirem menor número de sequências por segundo, porém essa se torna mais evidente na sequência 'pataka'. Além disso, para as crianças do GC houve correlação entre o número de sequências por segundo e a idade indicando que à medida que aumenta a maturidade aumenta a velocidade de produção de sequências.

As provas da DDK forneceram informações importantes para complementar o diagnóstico do TF, destacando-se que a produção articulatória de sílabas em sequência parece diferenciar melhor os grupos de sujeito do que a repetição de sílabas isoladas ${ }^{(21)}$ o que ressalta a influência da extensão da palavra no controle motor da fala.

Os resultados do presente trabalho incentivam novas pesquisas em que se verifique a interferência do tempo de produção na análise da DDK e do tipo de sequência mais indicada para o estudo de crianças com TF.

\section{CONCLUSÃO}

$\mathrm{Na}$ literatura, de forma geral, observa-se que os estudos sobre crianças com transtorno fonológico ainda estão muito voltados para a descrição da manifestação desta alteração. $\mathrm{O}$ presente estudo mostrou a importância de se avaliar funcionalmente, por meio da aplicação de diferentes métodos de análise, aspectos do processamento motor da fala em crianças com transtorno fonológico.

Apesar de ter sido observada correlação entre as medidas da DDK e a idade, não houve diferença entre os grupos indicando que na faixa etária estudada as crianças ainda estão aprimorando o desenvolvimento motor da fala e, por isso, não foi possível diferenciar crianças com e sem diagnóstico de TF nas sequências da prova de DDK.

$\mathrm{O}$ fato de não haver diferença entre os gêneros em cada um dos grupos sugere que na faixa etária estudada as crianças manifestam habilidades semelhantes, independentemente do gênero, no que diz respeito à repetição rápida das sequências da prova de DDK.

A concordância entre os dois métodos de análise indicou que a análise manual é uma opção tão confiável quanto à realizada por meio do software MSP.

\section{AGRADECIMENTOS}

Agradecemos à Fundação de Amparo à Pesquisa do estado de São Paulo (FAPESP), pelo apoio concedido para realização dessa pesquisa, sobre processo número 2008/57145- 2.

* HFW delineou a pesquisa, participou da coleta e da análise dos dados, conclusão dos achados e elaboração do artigo; LOPN contribuiu com a tabulação dos dados, conclusão dos achados e elaboração do artigo; RAR participou da coleta e análise dos dados e da elaboração do artigo; TFB colaborou com a coleta e tabulação dos dados e elaboração do artigo.

\section{REFERÊNCIAS}

1. Shriberg LD, Austin D, Lewis BA, McSweeny JL, Wilson DL. The speech disorders classification system (SDCS): extensions and lifespan reference data. J Speech Lang Hear Res. 1997;40(4):723-40.

2. Dodd B, McIntosh B. The input processing, cognitive linguistic and oromotor skills of children with speech difficulty. J Speech Lang Pathol. 2008;10(3):169-78.

3. Dodd B, McIntosh B. Two-year-old phonology: impact of intput, motor and cognitive abilities on development. J Child Lang. 2010 Nov;37(5):1027-46.

4. Castro MM, Wertzner, HF. Speech inconsistency index in brazilian portuguese-speaking children. Folia Phoniatr Logop. 2011;63(5):237-41.

5. Williams P, Stackhouse J. Diadochokinetic skills: normal and atypical performance in children aged 3-5 years. Int J Lang Commun Disord. 1998;33:481-6.

6. Williams P, Stackhouse J. Rate, accuracy and consistency: diadochokinetic performance of young, normally developing children. Clin Linguist Phon. 2000;14(4):267-93.

7. Prathanee B, Thanaviratananich S, Pongjanyakul A. Oral diadochokinetic rates for normal Thai children. Int J Lang Commun Disord. 2003 OctDec;38(4):417-28.

8. Gadesmann M, Miller N. Reliability of speech diadochokinetic tes measurement. Int J Lang Commun Disord. 2008 Jan-Feb;43(1):41-54.

9. Modolo, D. J. Diadococinesia oral e laríngea em crianças. [dissertação]. São Paulo: Faculdade de Odontologia de Bauru da Universidade de São Paulo; 2007.

10. Wertzner HF, Alves RR, Ramos ACO. Análise do desenvolvimento das habilidades diadococinéticas orais em crianças normais e com transtorno fonológico. Rev Soc Bras Fonoaudiol. 2008 Abr-Jun;13(2):136-42.

11. Folha GA, Felício CM. Relações entre idade, porcentagem de consoantes corretas e velocidades de fala. Pro Fono. 2009 Jan-Mar;21(1):39-44.

12. Andrade CRF, Queiróz DP, Sassi FC. Eletromiografia e diadococinesia: estudo com crianças fluentes e com gagueira. Pro Fono. 2010 AbrJun;22(2):77-82.

13. Modolo DJ, Berretin-Felix G, Genaro KF, Brasolotto AG. Oral and vocal fold diadochokinesis in children. Folia Phoniatr Logop. 2011;63:1-8.

14. Wang YT, Kent RD, Duffy JR, Thomas JE. Analysis of diadochokinesis in ataxic dysarthria using the motor speech profile program. Folia Phoniatr Logop. 2009;61(1):1-11. 
15. Alves RR. Diadococinesia oral em crianças com e sem transtorno fonológico [tese]. São Paulo: Faculdade de Filosofia, Letras e Ciências Humanas da Universidade de São Paulo; 2011.

16. Wertzner HF. Fonologia. In: Andrade CRF, Befi-Lopes DM, Fernandes FDM, Wertzner HF. ABFW: teste de linguagem infantil nas áreas de fonologia, vocabulário, fluência e pragmática. 2a ed. Barueri - SP: PróFono; 2004. p. 5-31.

17. Andrade CRF, Befi-Lopes DM, Fernandes FDM, Wertzner HF. ABFW: teste de linguagem infantil nas áreas de fonologia, vocabulário, fluência e pragmática. 2a ed. Barueri- SP: Pró-Fono; 2004.

18. Furnari, E. Esconde- Esconde. São Paulo: Ática; 1993.

19. KayPENTAX. Software Motor Speech Profile (MSP) Model 5141, software instruction manual. Lincoln Park, NJ: KayPentax; 2008.

20. Castro MM, Wertzner HF. Influence of sensory cues on the stimulability for liquid sounds in brazilian portuguese-speaking children. Folia Phoniatr Logop. 2009;61(5):283-7.

21. Kent RD, Kent JF, Rosenberk JC. Maximum performance tests of speech production. J Speech Lang Hear Disord. 1987 Nov;52:367-87.

22. Depret MMP. Análise da diadococinesia articulatória e laríngea em indivíduos com e sem transtornos neurológicos. [dissertação]. São Paulo: Universidade Federal de São Paulo; 2005.
23. Kent RD. Motor control: neurophysiology and functional development. In: Caruso AJ, Strand EA. Clinical management of motor speech disorders in children. New York: Thieme Medical Publishers, 1999. p. $29-71$.

24. Green JR, Moore CA, Higashikawa M, Steeve RW. The physiologic development of speech motor control: lip and jaw coordination. J Speech Lang Hear Res. 2000 Feb;43(1):239-55.

25. Green JR, Moore C, Reilly K. The sequencial development of jaw and lip control for speech. J. Seepch Lang Hear Res. 2002 Feb;45(1):66-79.

26. Nip IS, Green JR, Marx DB. Early speech motor development: cognitive and linguistic considerations. J Commun Disord. 2009 JulAug;42(4):286-98.

27. Smith A. Development of neural control of orofacial movements for speech. In: Hardcastle WJ, Laver J, Gibbon FE. The handbook of phonetic sciences. 2a. ed. Wiley-Blackwell, 2010. p. 251-96.

28. Smith A. Speech motor development: integrating muscles, movements, and linguistic units. J Commun Disord. 2006 Sep-Oct:39(5):331-49.

29. Wertzner HF, Amaro L, Scholz S. Indice de inteligibilidade e classificação perceptiva da inteligibilidade de fala no distúrbio fonológico. In: Anais do XI Congresso Brasileiro de Fonoaudiologia, 2003 out 01-07; Fortaleza. 